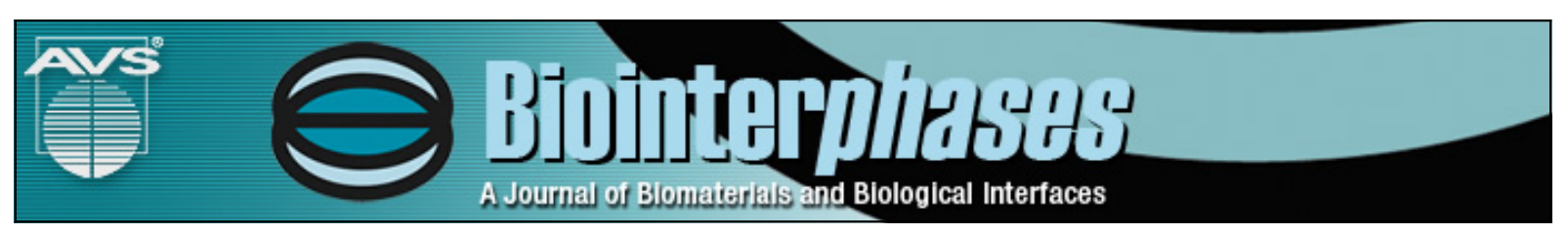

\title{
New strategies for developing cardiovascular stent surfaces with novel functions (Review)
}

Pengkai Qi, Si Chen, Tao Liu, Jialong Chen, Zhilu Yang, Yajun Weng, Junying Chen, Jin Wang, Manfred F. Maitz, and Nan Huang

Citation: Biointerphases 9, 029017 (2014); doi: 10.1116/1.4878719

View online: http://dx.doi.org/10.1116/1.4878719

View Table of Contents: http://scitation.aip.org/content/avs/journal/bip/9/2?ver=pdfcov

Published by the AVS: Science \& Technology of Materials, Interfaces, and Processing

\section{Articles you may be interested in}

A device for rapid and quantitative measurement of cardiac myocyte contractility

Rev. Sci. Instrum. 86, 034302 (2015); 10.1063/1.4915500

A finite element study of balloon expandable stent for plaque and arterial wall vulnerability assessment J. Appl. Phys. 116, 044701 (2014); 10.1063/1.4891019

Comparative study of CW, nanosecond- and femtosecond-pulsed laser microcutting of AZ31 magnesium alloy stents

Biointerphases 9, 029004 (2014); 10.1116/1.4866589

Controlled surface adsorption of fd filamentous phage by tuning of the $\mathrm{pH}$ and the functionalization of the surface J. Appl. Phys. 109, 064701 (2011); 10.1063/1.3549113

Announcement: A new feature-“Controversial Topics in Nonlinear Science: Is the Normal Heart Rate Chaotic?" Chaos 18, 030201 (2008); 10.1063/1.2960858 


\title{
New strategies for developing cardiovascular stent surfaces with novel functions (Review)
}

\author{
Pengkai Qi, Si Chen, and Tao Liu \\ Key Laboratory of Advanced Technology of Materials of Education Ministry, Southwest Jiaotong University, \\ Chengdu 610031, China and School of Material Science and Engineering, Southwest Jiaotong University, \\ Chengdu 610031, China \\ Jialong Chen \\ College of Stomatology, Anhui Medical University, Hefei 230032, China
}

Zhilu Yang, Yajun Weng, Junying Chen, and Jin Wang

Key Laboratory of Advanced Technology of Materials of Education Ministry, Southwest Jiaotong University, Chengdu 610031, China and School of Material Science and Engineering, Southwest Jiaotong University, Chengdu 610031, China

Manfred F. Maitz

Max Bergmann Center of Biomaterials Dresden, Leibniz Institute of Polymer Research Dresden, Dresden 01097, Germany

\author{
Nan Huang ${ }^{\text {a) }}$ \\ Key Laboratory of Advanced Technology of Materials of Education Ministry, Southwest Jiaotong University, \\ Chengdu 610031, China and School of Material Science and Engineering, Southwest Jiaotong University, \\ Chengdu 610031, China
}

(Received 22 March 2014; accepted 8 May 2014; published 21 May 2014)

\begin{abstract}
In this review, the authors summarize the developments in surface modification of cardiovascular materials especially in author's laboratory. The authors focus on three different strategies to construct multifunctional surfaces including coimmobilization of various biomolecules on stent surfaces, stem cell based therapy systems, and a single-molecule multipurpose modification strategy in vascular interventional therapy. The roles of various molecules like heparin, gallic acid, various aptamers, and nitric oxide are highlighted in the new strategies for developing cardiovascular stent surfaces with novel functions including excellent hemocompatibility, inhibiting smooth muscle cells proliferation, and native endothelium regeneration. The success of these multifunctional surfaces provides the tremendous potential in designing the next generation of vascular stents. (C) 2014 American Vacuum Society. [http://dx.doi.org/10.1116/1.4878719]
\end{abstract}

\section{INTRODUCTION}

The last half of the 20th century has witnessed the emergence of the medical device/implant industry. To our delight, the technological revolution in healthcare is growing rapidly, and most advances were in the area of cardiovascular technology. ${ }^{1}$ However, according to the statistics published by the world health organization in 2012, cardiovascular diseases (CVDs) continue to present the leading cause of death in modern society. ${ }^{2}$ The majority of the CVDs are related to a process called atherosclerosis, which is a chronic inflammatory disease marked by thickening, hardening, and stiffening of the arterial walls and results in impaired blood circulation. ${ }^{3}$ Severe impairment of the blood flow traditionally required intervention with bypass surgery. Vascular stents or grafts to treat these coronary and peripheral artery diseases emerged as minimal invasive alternatives to the surgery.

Coronary bypass surgery with patients' own saphenous vein or internal mammary artery has been the standard surgical treatment method to reestablish the blood flow in occluded arteries. ${ }^{4}$ Actually, there are difficulties in harvesting

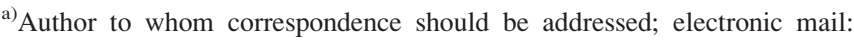
nhuang@263.net
}

sufficient lengths of autologous vascular grafts. Synthetic vascular grafts made of ePTFE (expanded polytetrafluoroethylene), PET (polyethylene terephthalate), PU (polyurethane), for example, are also used to replace or bypass diseased arteries. However, these materials, originally designed for industrial applications are associated with significant rates of restenosis and thrombosis. ${ }^{5}$ In addition to vascular grafts, coronary stenting has developed as a valid alternative method to prevent abrupt vessel closure and to achieve excellent angiographic results. Vascular stents are small expandable tubes, which are mounted onto a balloon catheter, inserted and expanded at the narrowed section of the vessel, acting like a stabilizing scaffold for the artery to maintain the patency of the vessel in order to treat narrowed or weakened arteries in the body. Despite of intensive research on improving vascular stents, failure cases of the implants still exist. In-stent restenosis (ISR), which results from intimal hyperplasia, frequently happened to patients implanted with the first generation of stents, the bare metal stents (BMS). ${ }^{6}$ Late stent thrombosis (LST) is a major complication of the patients who received implantation of the following generation of stents, the drug eluting stents (DES). ${ }^{7}$ Recently, new concepts of vascular stents like endothelial progenitor cell (EPC) capture stents and biodegradable stents (BDS) have emerged. In the clinical 
studies of the Genous Stent (anti-CD34 antibody coated surface, capturing EPCs), no acute or subacute stent thrombosis was observed. But CD34-positive cells can differentiate into various kinds of cells including inflammatory cells and vascular smooth muscle cells (SMCs) and thus these kinds of stents increased the risks of ISR and LST as well. ${ }^{8}$ Another promising approach to overcome these limitations is the application of biodegradable cardiovascular stents, which could be made of both polymers (lactic acid, ${ }^{9}$ glycolic, ${ }^{10}$ caprolactone families, ${ }^{11}$ etc.) and metals (Mg-based, ${ }^{12}$ Fe-based alloys, ${ }^{13} \mathrm{Zn}$ based, ${ }^{14}$ etc.). However, the degradation time of polymers of about $2 \mathrm{yr}$ is too long, and the uncontrollable degradation rate of magnesium within less than six month is too fast. There is still a gap between the degradation periods of existing stents and an ideal BDS degradation period, which is assumed to be about 6-12 months. ${ }^{15}$ Furthermore, the corrosion products, for example, lactic acid from poly(L-lactic acid) and $\mathrm{Mg}(\mathrm{OH})_{2}$ from magnesium alloys, significantly disturb the local environment by $p \mathrm{H}$ shifts, osmotic changes, and unphysiologic ion concentrations. Not all of these products underwent in depth evaluation yet.

Despite the impressive progress in the design of novel vascular stents, the pursuit of improving stent performance through surface modification has not stopped. From the BMS to DES, and from BDS to EPC capture stents, a large number of novel vascular stent models have been developed, focusing on new stent platforms, new bioactive coatings, novel functional surfaces, and new drug combinations. This article reports the recent research and applications of developing cardiovascular stent surfaces with novel functions emerged, especially in the author's laboratory. The objective of our work is to apply surface modification techniques for making cardiovascular stents to smart interventional devices, which can regulate blood-implant responses in a spatiotemporal way, accelerate the repair of endothelium and promote the healing of atherosclerosis.

\section{BASIC REQUIREMENTS OF AN IDEAL VASCULAR STENT}

Currently, numerous reviews have discussed the basic characteristics of vascular stents. Mechanical factors and biological factors are the main considerations in the design of a new stent. ${ }^{16}$ Mechanical factors include the following aspects: (1) both flexibility and strength are basic requirements to the substrate material. On one hand, it must be delivered to the small diameter atherosclerotic arteries; on the other hand, the substrate material should overcome the forces imposed by the vessel wall, avoid the recoil, and support vascular remodeling. That is why novel alloys, for example, Co-Cr and $\mathrm{Pt}-\mathrm{Cr}$ alloys, become popular instead of 316L stainless steel (SS). Hand in hand with the mechanical properties of the material goes the stent geometry, the thickness of the struts, the connections and the surface area. ${ }^{17}$ (2) Magnetic resonance imaging (MRI) compatibility: With the assistance of MRI, doctors are able to track the location of the stent and inflate the balloon through a catheter to expand the narrowed vessel wall. Taking Pt-Ir alloys for instance, it shows excellent radiopacity and also present a reduction in both thrombosis and neointimal proliferation. However, we have to face the problem of poor mechanical properties. ${ }^{18}$ Fortunately, with the development of material science and commercially available finite element solver, novel stent designs that match the substrate materials with the strut for better behavior has been a basic technique in almost all of the medical device companies.

However, it must be admitted that the advanced substrate materials only could provide a limited improvement of the stent performance. There are generally compromises between the optimized mechanical properties and the complex requirements of the bioenvironment. Consequently, sophisticated surface engineering has to be performed to address all the biological circumstances. ${ }^{19}$ (1) Hemocompatibility and antithrombotic properties: Materials surface should be blood compatible and able to resist plasma protein adsorption and denaturation, platelets adhesion and activation, blood coagulation factor activation, etc. (2) Suppression of SMCs: Excessive proliferation of SMCs is one of the initial factors in an atherosclerotic plaque. Also the SMCs associated neointimal hyperplasia is the main failure mechanism of BMS. (3) Endothelial cell-friendly microenvironment: Considering the significant role of the native endothelium in healing after the trauma of stent placement and as an antithrombotic surface, the ideal stent surface should actively promote endothelialization. ${ }^{20}$ Based on this point, the concept of EPC capture stents aiming at rapid endothelialization has raised up and plenty of animal experiments and clinical tests are under evaluation. The selectively targeted influence of the various vascular cells [platelets, SMCs, endothelial cells (ECs), EPCs] by the stent surface, however, is an ongoing and challenging task in surface engineering science.

\section{A. Surface functionalization strategies for improving hemocompatibility}

Blood-biomaterial interfacial events are considered as the key point when evaluating the efficiency of a stent. ${ }^{21}$ Immediately after exposure of the stent to the blood, plasma proteins adsorb to the surface of this foreign material and undergo conformational changes. Blood platelets respond to these external stimuli, especially to denatured fibrinogen on the surface by adhesion, aggregation, and conversion to a procoagulant state. In addition, the contact phase system (factor XII and kallikrein) of the intrinsic pathway coagulation cascade becomes activated, and the further progress of the coagulation is propagated by the activated platelets. ${ }^{22}$ Afterwards, the activation and aggregation of platelets and mononuclear cells and the generated fibrin form a stable thrombus and narrow the blood vessel further. ${ }^{23}$ From this point, limited hemocompatibility of DES substrate can lead to ISR after the degradation of drug-loaded coating and strongly contributes to the failure of these devices.

Surface modification techniques have been applied very early to improve the hemocompatibility of the stents. ${ }^{24}$ The 
key approaches to control blood component responses have been utilized to prepare surfaces with superior hemocompatibility, including inhibition of fibrinogen denaturation, inhibition of platelet aggregation and activation, blocking of the active catalytic site of thrombin, minimizing the complement associated inflammatory reactions, etc. ${ }^{25}$

\section{B. Surface functionalization for inhibiting smooth muscle cell proliferation}

Poor hemocompatibility is not the only reason for the failure of BMS. As stated above, ISR by neointimal hyperplasia is the leading complication of BMS. This intimal thickening, containing mainly SMCs, is a reaction of the vessel wall to the injury of stent expansion. The migrating SMCs cover the luminal surface of stent struts and often overgrowing them and cause the restenosis. Yet, the origin of the intimal SMCs remains controversial. ${ }^{26,27}$ In addition to the SMCs from the artery's media, also bone marrow derived smooth-muscle progenitor cells may participate in neointimal formation after vascular injury. ${ }^{28}$ It has been suggested that toxic ions released from the cobalt-alloy implants induce monocyte costimulatory molecule expression and release of proinflammatory cytokines and this may be the cause of the neointimal formation and the clinical failure of stents. ${ }^{29}$

For the prevention of ISR, a revolutionary stent design DES that combines drugs or agents with the medical devices has been developed. This kind of stent suppresses the development of lumen restenosis by the release of paclitaxel or sirolimus, both of which are antiproliferative drugs used in the treatment of cancer. Since its arrival in 2003, DES has transformed the practice of interventional cardiology by drastically reducing the restenosis rate and the need for revascularization. ${ }^{30}$ Unfortunately, these kinds of drugs also suppress the growth of ECs and delay the re-endothelialization. Besides, the polymer carrier like poly(ethylene-co-vinyl acetate) and poly(n-butyl methacrylate) in the first generation DES (Cypher ${ }^{\mathrm{TM}}$ and Taxus ${ }^{\mathrm{TM}}$ ), the delayed endothelialization also leads to extended exposure of the drug-eluting polymer coating to blood with the risk of LST. Nowadays, polymer-free DES and metallic DES with biodegradable polymers have been shown to improve the long-term safety and efficacy as well and new DES with thinner struts releasing limus-family analogues from durable polymers have further improved the clinical outcomes. ${ }^{31}$ However, also in the case of polymer-free DES and biodegradable carrier coated DES, a bare metal surface remains in contact with the vessel wall and/or the blood. The poor bio/blood compatibility of the bare substrate still can stimulate coagulation and cells dysfunction, especially soluble ions like $\mathrm{Cr}$, $\mathrm{Ni}$, and $\mathrm{Co}$ ions released into the tissue may induce adverse tissue reactions.

In order to overcome the shortcoming of the above mentioned stents, the corresponding author of this paper proposed the concept of a "time sequence functional stent," which combines a poly(lactic-co-glycolic acid) (PLGA)rapamycin coating on a $\mathrm{Ti}-\mathrm{O}$ coated metal stent substrate. The biodegradable PLGA controls the release of rapamycin and the subsequently exposed Ti-O film on the substrate presents good hemocompatibility. In the acute stage, the released drug plays a crucial role to suppress restenosis. Then the drug carrier PLGA starts to degrade and the Ti-O film with good thromboresistant properties is exposed to blood; it suppresses blood coagulation and supports endothelialization. ${ }^{32}$ In the late stage, the Ti-O film coating on the stent also prevents the release of toxic elements from the stent into the surrounding tissue and increases the long-term biocompatibility. One year clinical results showed that the restenosis rate and late thrombus events are significantly lower than the reference DES controls. ${ }^{33}$

\section{Construction of ECs friendly stent surface}

At present, DES are very popular and considered as a breakthrough in interventional cardiology. As a revolutionary medical device combining drug and stent, DES show statistically lower rate of ISR than BMS. ${ }^{34}$ However, the long-term safety of DES was questioned at the European Society of Cardiology Congress in 2006, and subsequent, studies reported that sirolimus or paclitaxel eluting stents were associated with similar risks of death or myocardial infarction as compared to $\mathrm{BMS}^{35}$ Late thrombosis has emerged as a major concern for DES implanted patients and those suffering from late DES thrombosis reminded us of the importance of arterial endothelium healing. The recent clinical results have elucidated that the complete endothelialization with ability of inhibiting SMCs' proliferation is an essential process for maintaining arterial health. The loss of endothelium is indeed a precondition for arterial thickening and restenosis. The drug released from DES inhibits both the growth of SMCs and ECs, slowing down the process of ISR but delaying the repair of endothelium. Actually, a healthy endothelium normally provides an anticoagulant and antiproliferative surface, acting as an effective natural protection against thrombosis. ${ }^{36}$

Nowadays, there is a development to design stent surfaces, which support endothelialization better. There is search for new drugs or molecules with selective cytotoxicity to SMCs but not to ECs for the application in DES. Apart from utilizing DES, improving stents without drug release, but stable modified with ligands for ECs adhesion molecules and EPCs capture molecule is another exciting and encouraging approach. Perhaps, future DES designs based on drug development will be guided more from the expertise of clinicians and pharmaceutical experts. Surface engineers are much more interested in the design of multifunctional surfaces which satisfy all the requirements of an ideal vascular stent to the greatest extent, typically via directing the cell development using bioactive molecules.

\section{NOVEL STRATEGIES IN CONSTRUCTING MULTIFUNCTIONAL STENT SYSTEM}

Since the success application of the Ti-O film based time sequence functional stents in our laboratory, we have paid more attention on new strategies for developing cardiovascular 
stent surfaces with novel functions in the recent years. In the following part, we would like to review our attempts for developing cardiovascular stent surfaces with novel functions, including our latest discoveries in three fields: (1) multifunction synergy by coimmobilization of biomolecules; (2) stem cell based strategies applied on vascular stents; (3) realization of multifuntionalization by single molecule based surface modification.

\section{A. Multifunction synergy by layer-by-layer coimmobilized biomolecules}

Multifunctional surfaces based on the coimmobilization of two or more biomolecules with synergistic action are frequently desired. At present, various techniques are available for immobilization of biomolecules on vascular stent surfaces including physical adsorption, monolayer self-assembly, and layer-by-layer (LBL), functional groups mediated anchoring and direct solution mixed covalent immobilization. The method of electrostatic LBL assembly with a cationic and an anionic polyelectrolyte, such as various growth factors and other proteins has been widely applied to construct a multifunctional surface. ${ }^{37}$ Aiming at constructing an anticoagulant, endothelialization supporting or EPC capturing surface, different polyelectrolytes, for example, heparin, adhesion proteins, and antibodies were chosen to construct the multifunction surface.

In the recent decade, many groups have adopted LBL to construct multifunctional surfaces. In the field of cardiovascular materials modification, this method is highly attractive, as it can be applied also to complex shaped surfaces. In 2009, Meng et al. alternatively deposited chitosan and heparin onto a coronary stent to accelerate the re-endothelialization and healing process after coronary stent deployment. The LBL technique was confirmed to be easily applied onto DES system and stable during sterilization and storage. ${ }^{38}$ With the same strategy, Luan et al. chose soluble complement receptor 1 and heparin as the assembled molecules to construct a surface that inhibits blood coagulation and complement activation and thus prolongs graft or stent survival. ${ }^{39}$ Recently, categories of assembling molecules have extended varying from the original proteins or charged polymers to novel drugloaded micelles or specific biomolecules like siRNA and antibodies. In 2009, Kim et al. reported a multilayer for stent system composed of anionic poly(lactic-co-glycolic acid) grafted hyaluronic acid micelles encapsulating paclitaxel, heparin, and PLL via an LBL fashion. The heparin and paclitaxel in suitable amount in the multilayer were readily controlled for a sustained release and effectively inhibited the proliferation of SMC. ${ }^{40}$ Lu et al. (2013) published their systematic results of ePTFE grafts coated with anti-CD133 functionalized heparin/collagen multilayers. Histopathological staining and scanning electron microscopy indicated that the anti-CD133 antibody accelerated the attachment of vascular endothelial cells onto the ePTFE grafts. They also emphasized that tailoring early rapid endothelialization had been of great significance in designing novel vascular grafts or stents. ${ }^{41}$ In the same year, hyaluronic acid/chitosan films with incorporated small interfering RNA (siRNA) nanoplexes were achieved by Wendel and Krastev groups. ${ }^{42}$ This stent surface suggested the low adhesion of blood cells on the polyelectrolyte multilayers, as it prevents thrombosis after stenting. Gene eluting system might offer a powerful technique for novel DES to prevent restenosis in the future. ${ }^{43}$ Fortunately, fabrication of multilayers by consecutive adsorption of polyanions and polycations provides a flexible platform for alternative deposition of novel biomolecules for tailored multifunction architecture.

Our group has been working on the construction of multifunctional stent surfaces via LBL techniques for a few years. Li et al. improved blood compatibility and endothelialization utilizing heparin and fibronectin (Hep/Fn) LBL coimmobilization. The effect of different $p \mathrm{H}$ conditions on the assembling effect depending was investigated and compared with covalent immobilization methods. All results suggested that the coimmobilized films of Hep/Fn confer good antithrombotic properties and with good endothelialization. ${ }^{44,45}$ Also, inflammatory responses of these modified samples were further evaluated. The results showed that Hep/Fn modified titanium revealed a lower inflammatory response than pristine $\mathrm{Ti}$, as determined by the release of the TNF- $\alpha, \mathrm{MCP}-1$ and IL- $1 \beta$ from macrophages and endothelial cells seeded onto the materials. ${ }^{46}$ Also extracellular matrix components such as collagen, ${ }^{47}$ fibronectin, ${ }^{48}$ laminin and their derived peptide sequences RGD, REDV, YIGSR, etc., were integrated in together with heparin in LBL deposited coatings on titanium surfaces, and these surfaces were shown to improve EC adhesion and proliferation and enhance the hemocompatibility. However, the detailed mechanism of multifunctional synergy of heparin and adhesive proteins remains unclear, which still needs further investigation.

\section{B. Stem cell based strategies applied on vascular stents}

A novel strategy of in vivo/in situ endothelialization of vascular implant for accelerating vessel repair after stent implantation has been recently developed. Supporting the homing of stem cells or progenitor cells at the site of the injury, accelerates endothelialization and presents a self-healing system with accelerated repair of the damaged endothelium. ${ }^{49}$ The responsible cell source for re-endothelialization, the EPCs has been first described by Asahara in $1997 .{ }^{50}$ In the recent decades, this small population of $\mathrm{CD} 34^{+}$circulating mononuclear cells derived from bone morrow gained increasing interest, because of its capability to differentiate into mature ECs and participate in therapeutic angiogenesis in vivo.

\section{Stem cell capture surface for rapid endothelialization}

In 2005, Genous stent received CE mark and became the first commercial vascular stent applying an EPC-capture system based on immobilized anti-CD34 antibodies. ${ }^{51}$ Despite 
absence of acute or subacute stent thrombosis or stenosis in clinical trials, there appeared the possibility that the stent also pilots smooth muscle progenitor cells, which cause neointimal proliferation. Continuous improve of EPC capture stents therefore is still a huge task for the stent designers. Lin et al. applied LBL techniques to construct a coated antiCD34 functionalized heparin-collagen multilayer with good results concerning in situ endothelialization and as a possible solution against in-stent restenosis. ${ }^{52}$ Anti-CD34 antibodies also were grafted on a titanium surface via oriented immobilization techniques. ${ }^{53}$ This coated surface could promoted EPC adhesion under static and flowing condition in vitro, and induced rapid endothelialization of a stent in vivo. Although these designs could ensure a sufficient amount of CD34 ${ }^{+}$stem cells, the cells did not differentiate further to endothelium. Taking this limitation into account, heparin, CD34 antibody and vascular endothelium growth factor (VEGF) were coimmobilized on the stent surface, striking the balance of antithrombosis and effective ECs-promotion. ${ }^{54}$ As shown in Fig. 1, avidin was absorbed onto titanium via electrostatic interactions. Afterwards, biotinylated heparin and Protein A were immobilized onto the avidin coated surface through biotin-avidin system. Subsequently this layer was used to immobilize the anti-CD34 antibody and VEGF, respectively, which is based on the specific binding of protein A and Fc segments of CD34 antibody and the specific affinity of heparin and VEGF. It was concluded that a biofunctional LBL on titanium, constructed by assembling the anti-CD34 antibody, VEGF, and heparin, significantly improves the endothelialization and anticoagulation.

Even though these designs proved to be safe and effective, the success of this fascinating technique largely depends on the selection of optimal target molecules on the membrane of the EPC surface. Wendel et al. questioned that the concept of EPC capture stents is hype or hope. The challenge is if the capture molecules bind even weakly to other blood cells (e.g., platelets, monocytes, and neutrophils) or plasma proteins (fibrinogen, etc.), this technique will fail. The immobilized capture molecules will be immediately covered by competing proteins or cells considering their significantly higher concentration in the blood stream than EPCs. ${ }^{55}$ In literature, numerous molecules for capturing EPCs have been applied to achieve self-endothelialization of blood-contacting materials including monoclonal antibodies, selectins and their ligands, peptide or DNA aptamers, and even magnetic molecules. ${ }^{56}$ In 2013 , Lee et al. compared endothelialization and neointima formation of two EPC capture stents with $\mathrm{CD}_{3} 4^{+}$antibodies or vascular endothelialcadherin, respectively, coated on BMS. The VE-cadherin worked out as the more suitable target molecule than CD34 for EPC-capturing stents. ${ }^{57}$ Our group also has made attempts on constructing EPC capture surface. Using the
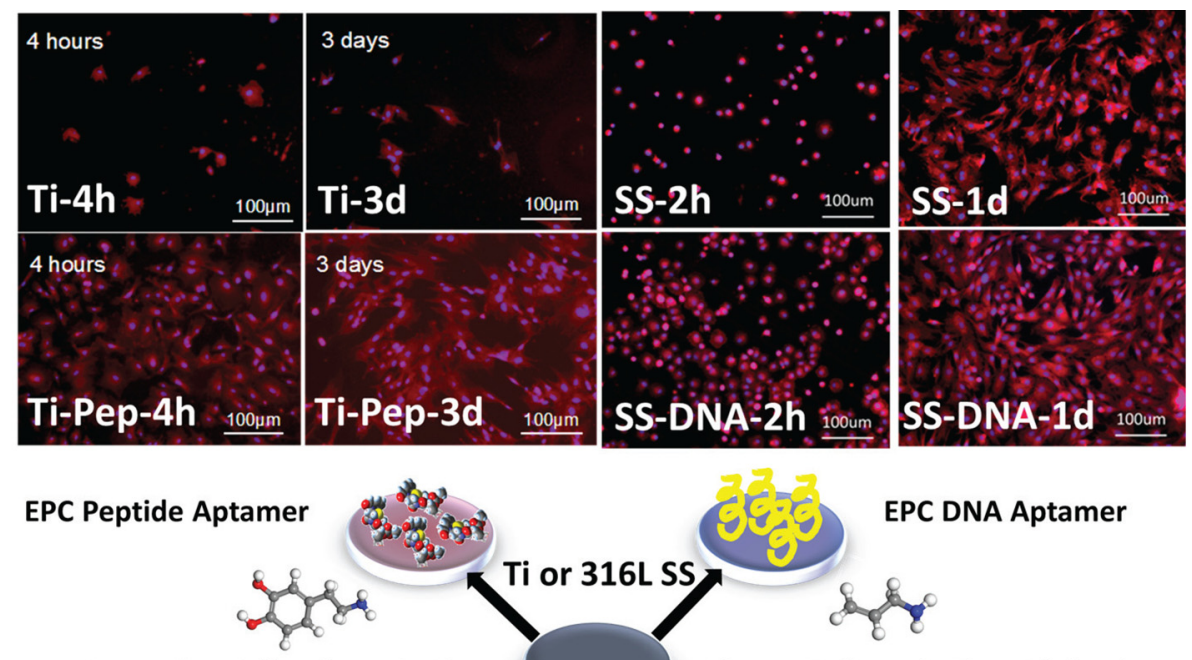

Dopamine Self-polymerization
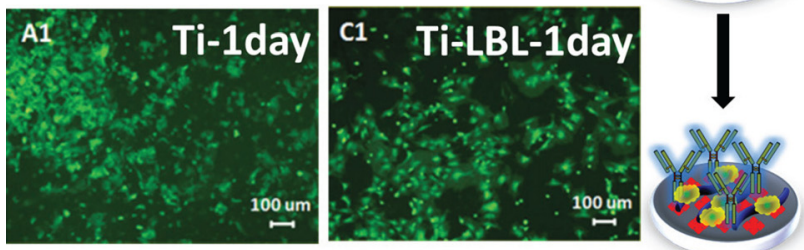

Plasma Polymerization Allylamine

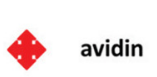

Biotinylated heparin * VEGF
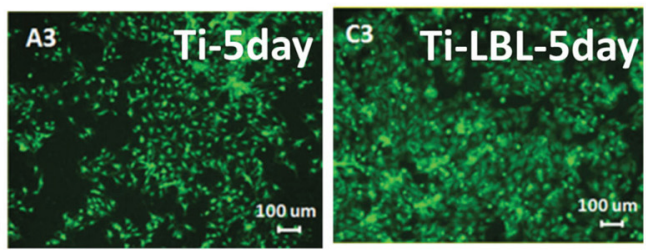

Antibody CD34

FIG. 1. Fluorescent images of EPCs, which adhered under flow conditions on bare titanium (Ti) or SS surface, or on these metals functionalized with EPCcapture peptide- or DNA-aptamers or anti-CD34 antibodies. The peptide aptamer was grafted via Schiff's base reaction on a polydopamine coating, the DNA aptamer via electrostatic adsorption onto a plasma polymerized allylamine coating and the antibody together with heparin and VEGF in an electrostatic layerby-layer deposition technique. Cell nuclei are stained blue (DAPI) and cytoplasma in green/red (Rhodamine). 
biotin-avidin system, a peptide aptamer(TPSLEQRTVYAKGGGC-K-Biotin) and bovine serum albumin (BSA) were immobilized onto titanium via mussel-inspired dopamine film as shown in Fig. 1 (unpublished data). In vivo experiments showed that the aptamer and BSA coimmobilized surface had only low antithrombogenic property but also accelerated the self-endothelialization in situ. Another trial was the immobilization of DNA aptamers to construct an EPC-capture surface on a plasma polymerized allylamine (PPAam) film. The modified samples captured more EPC under a nonstatic conditions and present a cell friendly surface for both EPCs' and ECs' proliferation (Fig. 1, unpublished data). However, there are also limitations with this design. Peptide or DNA aptamers that specifically bind to EPC were obtained through an in vitro selection process known as phase display ${ }^{58}$ and systematic evolution of ligands by exponential enrichment, ${ }^{59}$ respectively. Their biosafety and biostability are still unknown because in vivo animal tests and long term evaluation have not been conducted systematically. However, taking advantages of EPC-specific molecules to utilize the self-healing system of the organism by fishing EPC to the vascular lesion have brought a paradigm change in surface modification concepts for in vivo endothelialization. Our group continuously performs more basic studies and animal tests to optimize the EPC capture surface in order to develop a novel generation vascular stent based on this principle.

\section{Stem cells mediated vessel healing in multiple dimensions}

EPCs can be isolated and labeled by incubation with superparamagnetic iron oxide (SPIO) particles via phagocytosis processes. This method to capture and to retain mesenchymal stem cells or circulating EPCs at the vascular injury site provides a novel approach to realize a targeted guidance of the cells. Li et al. labeled EPCs with silica coated SPIO nanoparticles and an applied exterior magnetic field to guide it toward the ischemic region in a mouse model of middle cerebral artery occlusion in vivo. ${ }^{60}$ Riegler et al. also demonstrated that conjugation with SPIO nanoparticles does not exhibit adverse effects on cell viability, differentiation, or secretion patterns. The increase in stem cell retention resulted in a decrease in the restenosis level three weeks after cell delivery. ${ }^{61}$ Hence, our group prepared polyethylene glycol (PEG) and anti-CD34-coated magnetic nanoparticles (MNPs) to guide stem cells to a target destination in vivo by MNPs in a magnetic field. Hemolysis rate and clotting time (APTT) were determined to ensure the safety of the application in the cardiovascular system. The ability of nanoparticles to target stem cells in vitro and the influence of the magnetic field strength on the stem cells capture effect were evaluated as well. The designed MNPs possessed good hemocompatibility and specifically bound to stem cells (Fig. 2). The suitable response to an external magnetic field helped to deliver stem cells to the lesion sites. ${ }^{62}$ Inspired from Polyak group's work, ${ }^{63}$ our synthetic magnetic nanoparticles were applied to construct an EPC captured surface under exterior magnetic field. Different from EPCbinding device surfaces, CD34 ${ }^{+}$binding MNPs can harvest enough EPCs from the whole blood and guide these cells to the location of the stent implantation by an external magnetic field.

With the development of biodegradable vascular stents, Fe-based alloys also gained interest. ${ }^{13}$ However, the low corrosion rate and difficulty in biological surface modification limit the application of these materials in vascular stent design. ${ }^{15}$ MNP guidance of EPCs, the magnetic iron stent may find a revival in the future. Rapid endothelialization on an iron stent surface may be accomplished under a magnetic field. MNP-labeled stem cells can be accumulated at the magnetic iron surface to achieve a native endothelium layer. At various time points, repeatedly fresh MNPs can be injected into the body to capture regenerated stem cells to the desired location. From this conceive, stem cells mediated injured vessel healing in multiple dimensions (time-space relationship) is achieved as a more efficient treatment.

\section{Multifuntionalization by single molecule based surface modification}

Currently, multifunction surfaces become the goal of novel stent designs. Nevertheless, realization of multifunction requires the cooperative work of multiple molecules. More extended clinical evaluation of isolated and combined hazards to the patients are necessary with these more complex devices. Therefore, a single molecule with multiple functions, i.e., endothelialization promotion, anticoagulant, antiplatelet, SMC suppression, and remedying atherosclerosis, for immobilization on the stent surface was sought. During few years' exploration in our group, several promising molecules were identified and immobilized. For instance, heparin, GA, and nitric oxide (NO) donor systems have been successfully introduced.

\section{Heparin associated multifunctional stent coatings}

Heparin, a highly sulfated polysaccharide, was discovered in $1959 .{ }^{64}$ It is known as an anticoagulant drug and interacts with a wide range of proteins, chemokines, and growth factors, and these interactions are implicated in immunology, physiological, pathological processes, and many other biological processes, such as tissue structuring and organization, blood clotting, and cell growth. ${ }^{65}$ Figure 3 reveals the multiple functions of heparin. As an anticoagulant polysaccharide, heparin of 18 or greater saccharide units catalyzes the interaction of antithrombin and thrombin, leading to the formation of covalent thrombin-antithrombin complexes, and thus exhibits anticoagulant activities. A pentasaccharide sequence of heparin already is sufficient to inhibit the coagulation factor Xa. This knowledge has been used for long time to produce anticoagulant coatings on surfaces by immobilizing heparin at high concentrations to a surface using various methods, ${ }^{66}$ up to the recent development of feedbackcontrolled, coagulation triggered heparin release systems. ${ }^{67}$ 


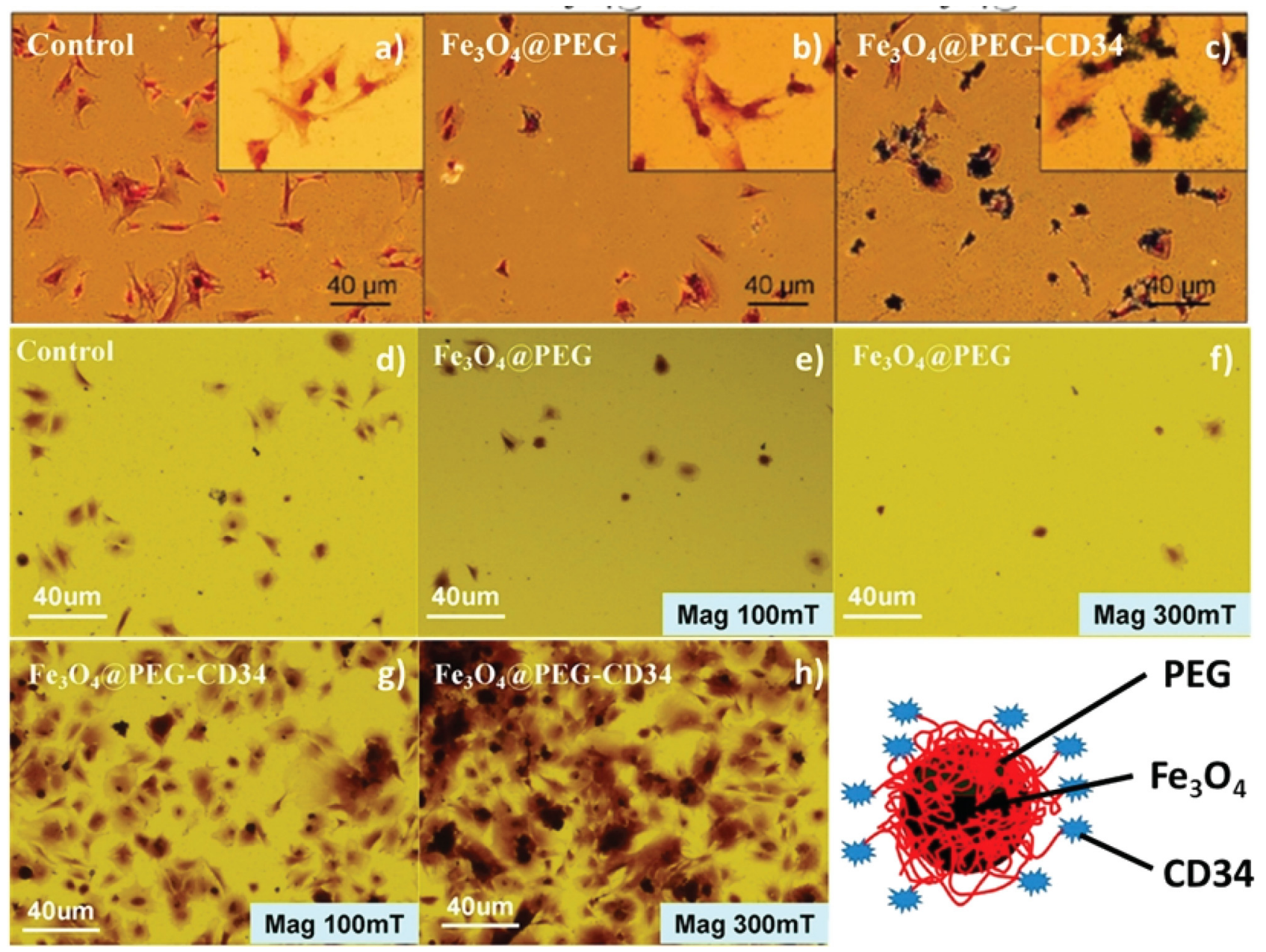

FIG. 2. Prussian blue stained stem cells incubated with different magnetic nanoparticles for one day. Incubation without magnetic field in (a)-(c). Incubation for two days under the different magnetic field intensities of $100 \mathrm{mT}$ in (e), (g), and $300 \mathrm{mT}$ in (f), (h), (d) without magnetic field as control.

Besides this, heparin inhibits platelet function and induces the expression of markers of contractile SMC phenotype and thus reduces SMC proliferation, which is considered as the major mechanism by which local heparin therapy inhibits restenosis. ${ }^{68}$ Heparin also presents anti-inflammatory properties by inhibition of the complement cascade and promotes endothelium regeneration in some cases. The possible principle is the high affinity of various growth factors and other proteins with an anion binding site to heparin. However, the mechanism by which heparin promotes human endothelial cell growth is not clear yet.

In our group, a heparinized surface was constructed via covalently immobilization or ionic adsorption to a PPAam film to explore the role of the heparin immobilized surfaces in the direction of ECs and SMCs fate and re-endothelialization. ${ }^{69}$ The results [Fig. 4(a)] suggest that ECs on the Heparin-PPAam undergo the general adhesion processes of substrate attachment, spreading, and cytoskeleton development and then proliferation. In addition to EC, also the morphology, proliferation, and viability of SMCs were analyzed. The Heparin-PPAam surface can effectively inhibit cell adhesion, survival, proliferation, and increase the level of SMCs apoptosis [Fig. 4(b)]. We also noticed that the PPAam surface covalently immobilized with $3.6 \mu \mathrm{g} / \mathrm{cm}^{2}$ heparin exhibited reduced adhesion and proliferation of ECs compared to bare PPAam but higher adhesion and proliferation of ECs than on 316L SS. PPAam functionalized with only $0.28 \mu \mathrm{g} / \mathrm{cm}^{2}$ heparin in a noncovalent manner showed much greater ability to enhance EC adhesion and proliferation than the $316 \mathrm{~L} \mathrm{SS}$ as well as the control PPAam. ${ }^{70}$ Another heparinized surface was constructed in our group by immobilizing heparin/poly-L-lysine nanoparticles conjugated at different $p \mathrm{H}$ onto a dopamine coated surface, where a heparin density gradient on a surface was generated by immobilizing nanoparticles of different heparin: poly-L-lysine ratios. ${ }^{71}$ The AT III binding quantity on this surface was significantly enhanced, and APTT and TT coagulation times were prolonged, depending on the heparin density. Also, a low heparin density is sufficient to prevent platelet adhesion and activation. Meanwhile, the biological compatibility evaluation suggested that a high heparin density above $20 \mu \mathrm{g} / \mathrm{cm}^{2}$ was unsuitable for vascular cell proliferation and endothelium regeneration, while low heparin density up to $3.5 \mu \mathrm{g} / \mathrm{cm}^{2}$ selectively prevented SMCs proliferation but accelerated endothelialization. Therefore, the sensitivity of vascular cells to the heparin density is very different, high 


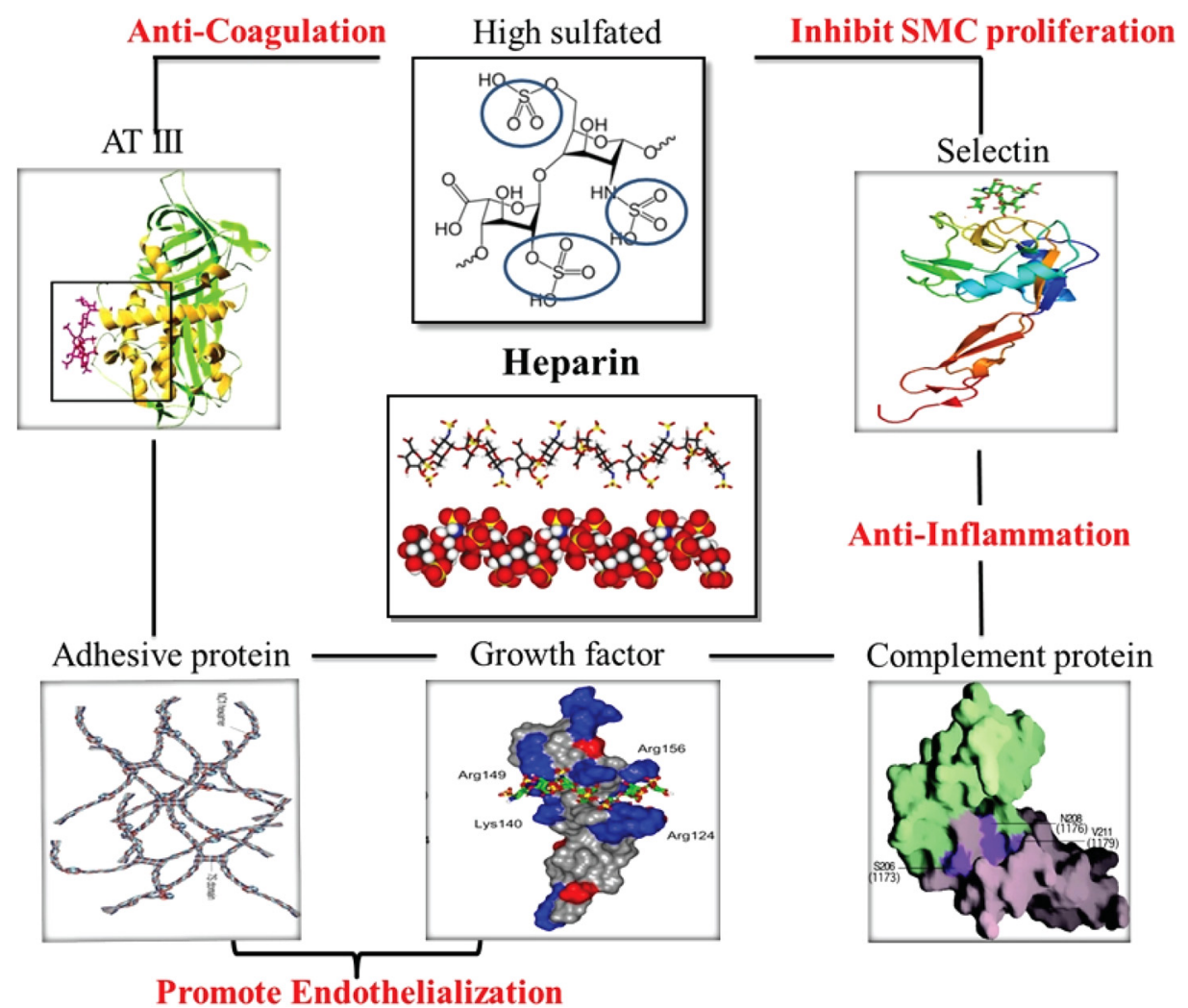

FIG. 3. Multiple functions of heparin and associated biomolecules.

A
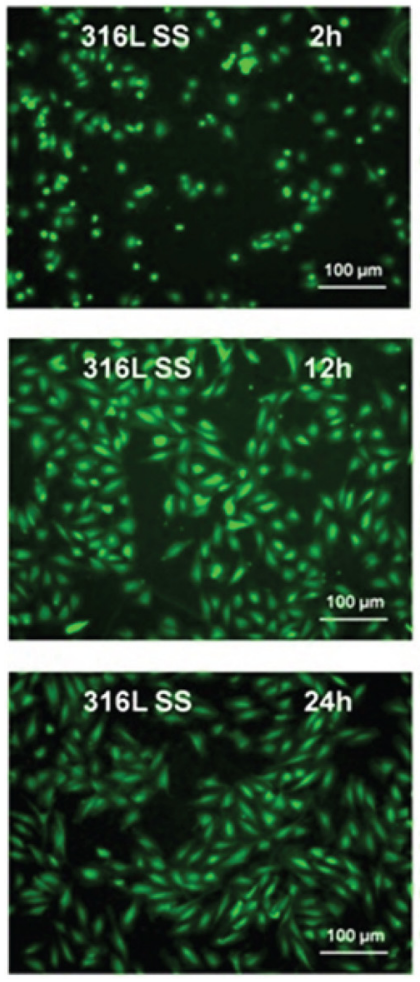

B
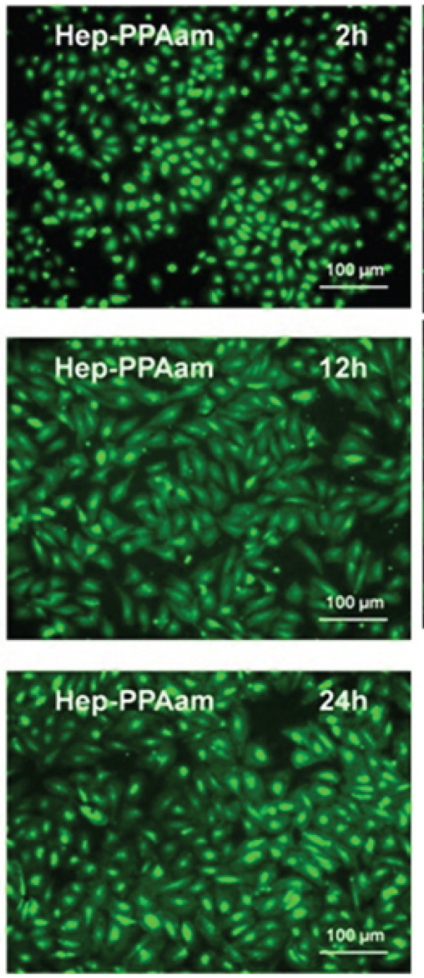

B
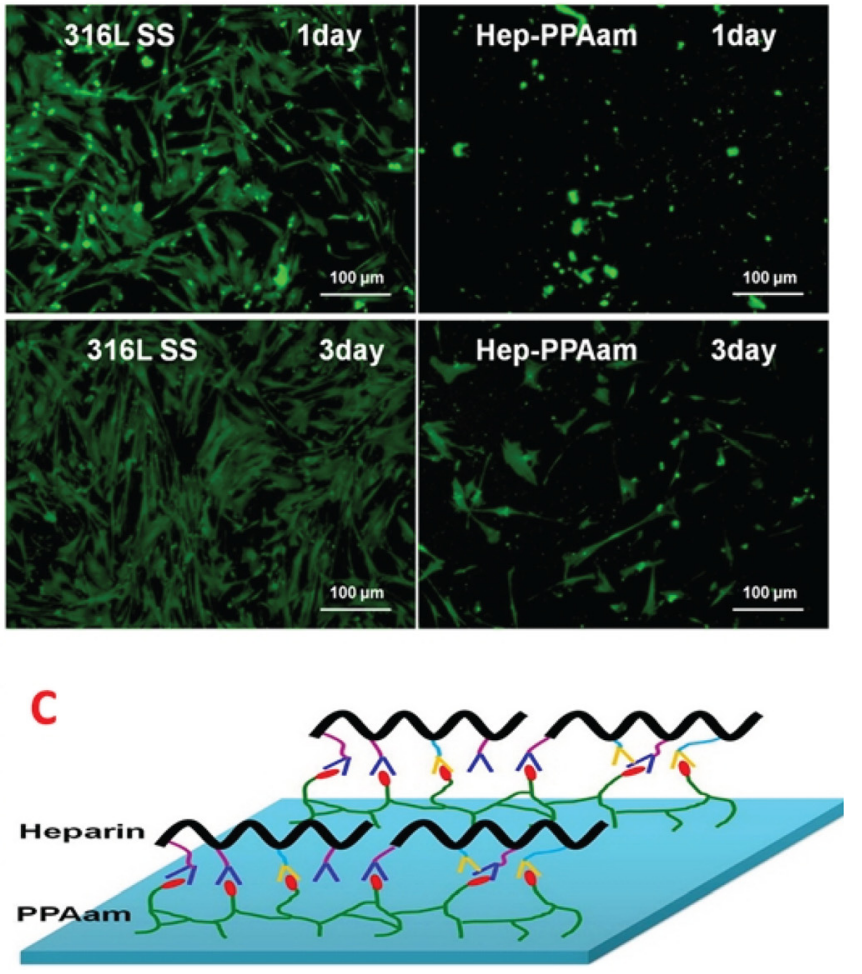

FIG. 4. (a) Immunofluorescent images of cytoskeletal actin for ECs on the 316L SS and Hep-PPAam surfaces after 2, $12 \mathrm{~h}$, and $24 \mathrm{~h}$ of culture. (b) Immunofluorescent images of $\alpha$-SMA for HUASMCs on 316 L SS, Hep-PPAam after one and three days of culture. (c) Schematic diagram of heparin adsorption on the protonated PPAam surface. 
heparin density suppresses the growth of all vascular cells, while low heparin density selectively inhibits SMCs hyperplasia but promotes EPCs and ECs proliferation.

According to others' previous report on heparinized surfaces, for example, heparin-coated stent of Gong et al. ${ }^{72}$ and heparin-doped polypyrrole by Stewart et al., ${ }^{73}$ we conclude that a material decorated with heparin, either bound in a covalent or noncovalent manner, exhibits multiple functions depending on the exposed heparin density. These results of basic research promise a successful construction of multifunctional heparinized surface which may reduce restenosis and promote endothelialization, providing further concepts to utilize heparin more effectively on surface modification of vascular stents.

\section{Gallic acid assisted surface modification of vascular stents}

GA (3,4,5-triphydroxyl-benzoic acid) is particularly abundant in processed beverages such as red wines and green teas. It is a polyphenyl natural product, which can be obtained under acid hydrolysis of hydrolysable tannins. ${ }^{74} \mathrm{It}$ is a strong natural antioxidant and presents some therapeutic properties including antitumoral, antibacterial, antiviral, and anti-inflammatory activities as observed in previous studies. $^{75}$ In addition, GA has been found to selectively induce apoptotic cell death in SMCs but no cytotoxicity against normal ECs. ${ }^{76}$ Based on these considerations, we introduced GA in multifunctional films in various studies.

Taking the pharmacological effect of GA into account, the influence of different concentrations of GA on ECs and SMCs was investigated through culture of single cell types and coculture, and some systematic results are shown in Fig. 5. The ratio of ECs to SMCs in coculture condition has a significant rise for GA concentrations of $5 \mu \mathrm{g} / \mathrm{ml}$. These data indicate that GA exhibits a selective dose-dependent effect on ECs and SMCs, which promotes the growth of ECs, while inhibits the growth of SMCs. For the design of novel DES, GA with a released concentration of $5 \mu \mathrm{g} / \mathrm{ml}$ may be a suitable candidate to be loaded in a polymer coating. ${ }^{77}$ Another distinguishing feature of GA is the content of catechol groups which can adsorb on metal surfaces. Inspired of mussel derived dopamine self-polymerization, GA, and hexamethylenediamine (HD) were used to form a film (GAHD) under alkaline conditions (Tris $10 \mathrm{mM}$, $p \mathrm{H}=8.5$ ). The possible copolymerization mechanism of GA and HD has been elucidated and results of biocompatibility evaluation of GAHD film have been reported in 2013 (Fig. $6)$. It shows the immobilization strategy of heparin, laminin, or fibronectin on the film with the coexistence of multiple functional groups. ${ }^{78}$ Except of these adhesive proteins, antiCD34 antibodies and VEGF were also coimmobilized onto the GA modified PPAam surface. Cell culture results indicated significant enhancement in capturing EPCs and the proliferation of ECs. ${ }^{79}$ To our delight, the GA-involved multifunctional coating shows a huge potential in tailoring the desired interfacial properties onto a wide range of materials. It selectively induces SMCs apoptosis but supports ECs growth, suggesting a promising application for the design of an ideal multifunctional vascular stent that is able to address the issues of re-endothelialization and restenosis. ${ }^{80}$

\section{Nitric oxide signal mediated stent design with multifunctional surface}

NO is a signaling molecule that plays a pivotal role in the diagnosis, treatment, and prevention of life threatening cardiovascular disorders such as coronary artery disease, essential hypertension, stroke, vascular complications of diabetes, and related diseases. ${ }^{81}$ This cell signaling molecule that was first discovered in the 1980s and once as endotheliumderived relaxing factor it is constitutively and inducibly expressed from ECs through enzymatic conversion of L-arginine by various nitric oxide synthases (NOS). ${ }^{82}$ It has been the subject of significant research not only in the basic sciences, but also in applied sciences such as the biomaterials field. ${ }^{83,84}$ As a star molecule in the cardiovascular system, NO released from healthy endothelium simultaneously possesses anticoagulant, ${ }^{85}$ antiproliferative ${ }^{86}$ properties and has the ability to promote the regeneration of healthy endothelium $^{87}$ (Fig. 7). Even in other cases, NO-delivery platform present antibacterial properties ${ }^{88}$ and accelerate wound
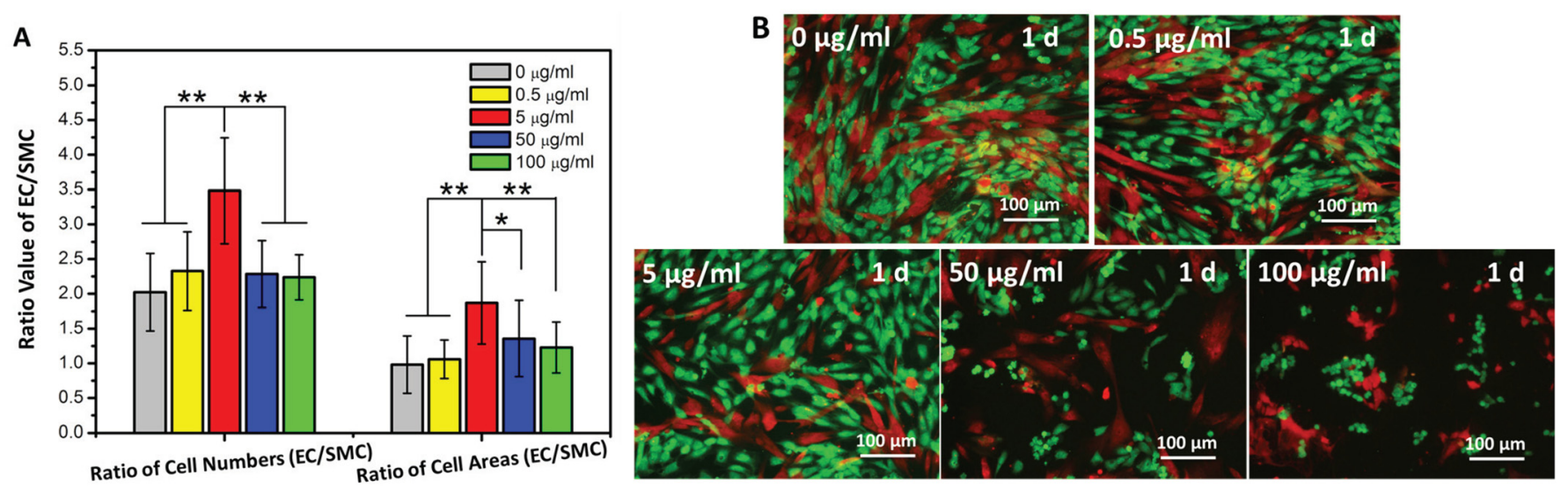

FIG. 5. (a) Ratio of ECs and SMCs in cell numbers and cell area coverage in coculture after one day in medium containing GA. (b) Fluorescence micrographs of EC (green) and SMC (orange). Scale bar is $100 \mu \mathrm{m}$. 


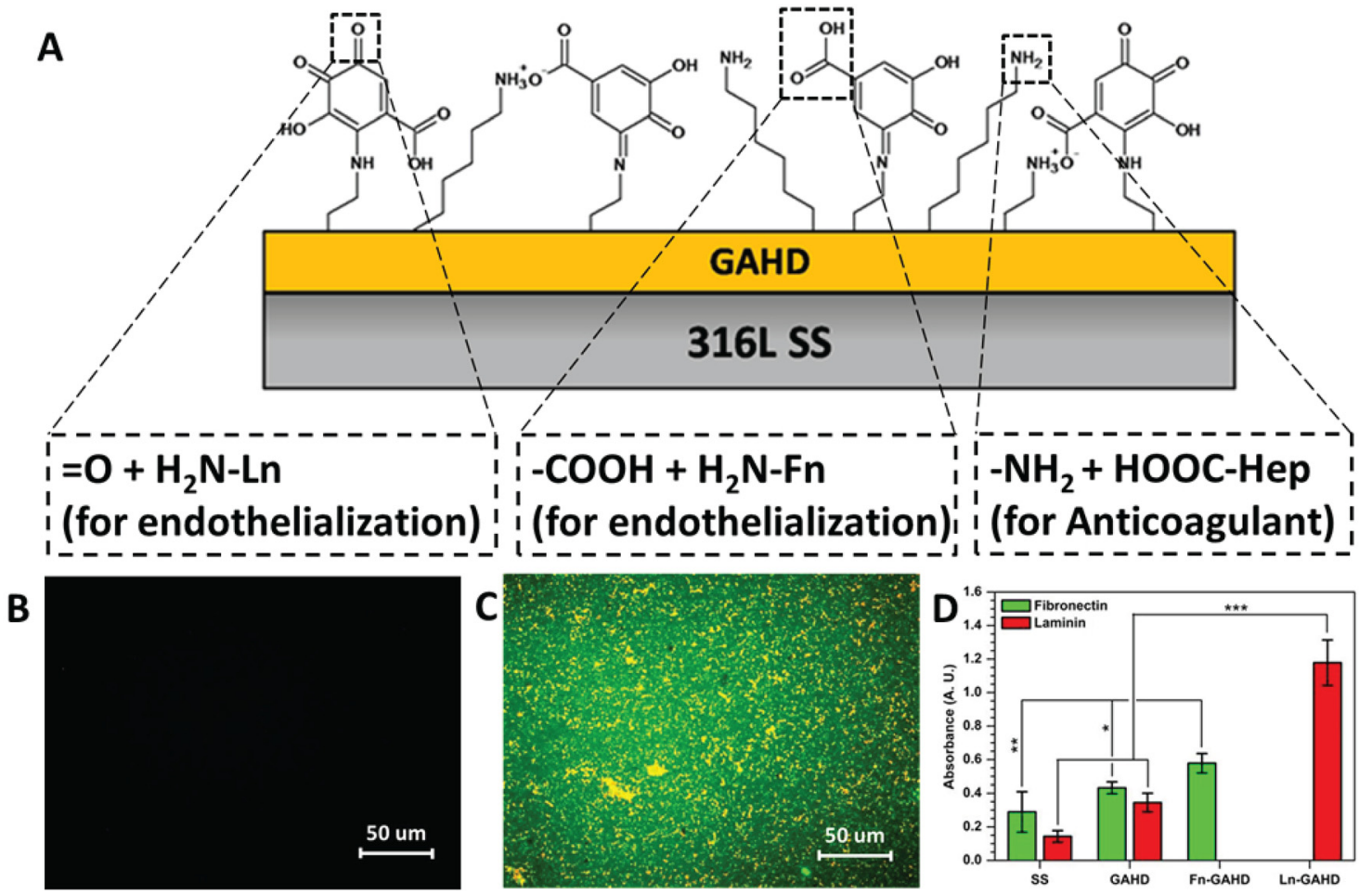

FIG. 6. (a) Chemical structures and functional groups on polymerized GA and HD films. B) Fluorescence image of GAHD (blank). (c) Distribution of laminin (green) and fibronectin (red) coconjugated to the GAHD surface via Schiff base reaction and carbodiimide chemistry, respectively. (d) Quantization of Fn and Ln immobilized on Fn-GAHD and Ln-GAHD samples.

healing process. ${ }^{89}$ Also, it plays a critical role in regulating immune responses ${ }^{90}$ and inducing differentiation of stem cells. ${ }^{91}$ These unique advantages make $\mathrm{NO}$ a very promising molecule in the design of an ideal vascular stent.

Over the past few years, researchers (typically Meyerhoff group) have been devoting themselves on the development of efficient NO-releasing and -generating materials for clinical therapies. Unique strategies for designing NO releasing materials have been widely reviewed. ${ }^{92}$ Micelle, nanoparticles, or dendrimers were synthesized with NO donors such as S-nitrosothiols. Polysaccharide-based, metal complexbased, heparin combined, and adhesive protein-combined surface were also reported. These NO-donors type, particletype, and coating platforms present multiple biological functions. However, there still exist some limitations of this kind of material. A great challenge lies in the fabrication of cardiovascular biomaterials while accommodating NO donors due to their reactivity, nature, instability during storage, and leaching of toxic byproducts. ${ }^{93}$ To overcome the present limitations, recent strategies focus on transition metal-ions

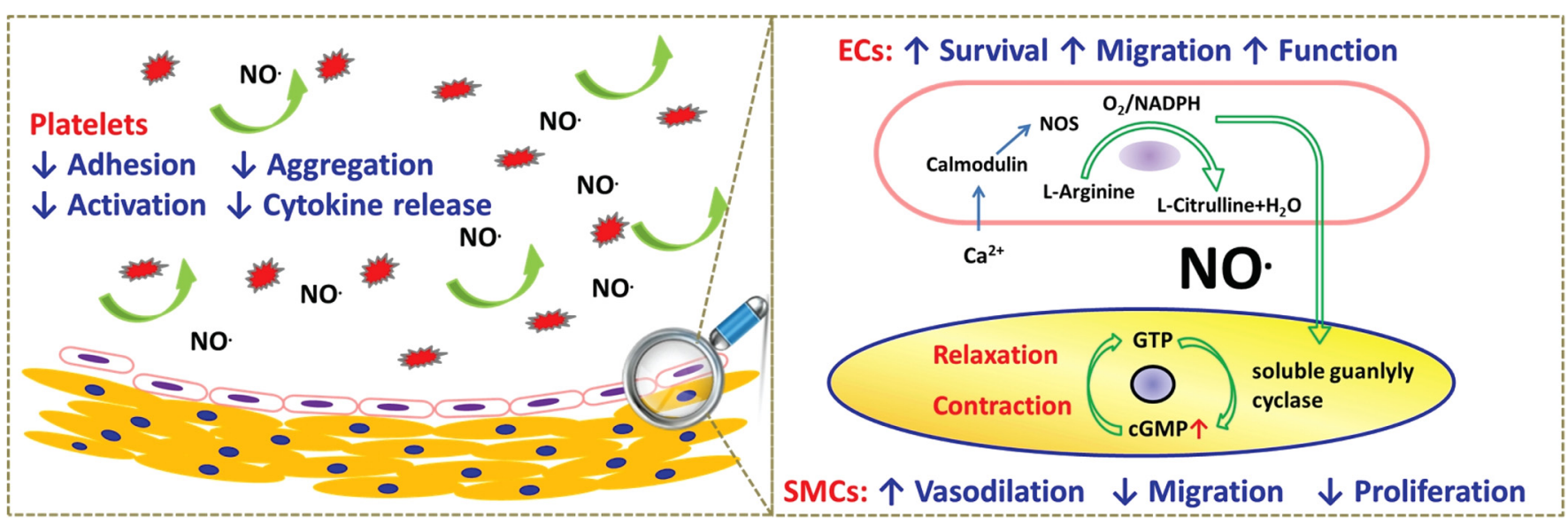


mediated catalysis $\left(\mathrm{Cu}^{2+}, \mathrm{Fe}^{2+}, \mathrm{Hg}^{2+}, \mathrm{Ag}^{+}\right.$, organoselenium/tellurium compounds, or cystamine) to generate NO from endogenous NO donor-RSNOs via specific glutathione peroxidase(GPx)-like catalytic activity reactions. ${ }^{94,95}$ The catalytic NO generation strategy was adopted to construct multifunctional surfaces. The main reason is the expected long-term release of NO via an endogenous NO donor improves the stent performance.

In 2010, our group has taken an attempt on immobilizing selenocystamine onto the Ti-O film to prepare a NOgeneration surface. ${ }^{96}$ Briefly, Ti-O was deposited onto $316 \mathrm{~L}$ SS via unbalanced magnetron sputtering. Multilayers of polydopamine were grafted to the $\mathrm{TiO}_{2}$ surface by immersion of the substrates in a $2 \mathrm{mg} / \mathrm{ml}$ dopamine solution. The subsequent oxidation process depends on the reaction of amine groups of the selenocystamine(SeCys) via schiff base reaction and Michael addition [Fig. 8(a)]. This SeCys modified surface presents GPx-like activity and catalytically generates NO with a rate of about $1 \times 10^{-10} \mathrm{~mol} \mathrm{~cm}{ }^{-2} \mathrm{~min}^{-1}$, which approaches the normal NO synthesis and release from healthy endothelial cells. ${ }^{97}$ Through the release of NO, the modified surface remarkably inhibited the adhesion of platelets and SMCs. In addition, it inhibited collagen-induced platelet activation and aggregation. The increase of the intracellular cGMP concentration confirmed that these phenomena were due to NO specific signaling in platelets and SMCs [Figs. 8(b) and 8(c)]. SeCys immobilized stents were implanted into canine femoral arteries and the two months in vivo implantation results showed significantly inhibited intimal hyperplasia [Fig. 8(d)], suggesting the great promise of the NO-releasing stent surface for cardiovascular therapy. The NO-generating coatings therefore are also very promising candidates for a new generation of stents due to their versatile abilities to improve hemocompatibility, enhance endothelialization, inhibit SMC proliferation combined with the potential biological functions of NO generation in immune response, antibacterial behavior, inflammation prevention, and healing atherosclerosis. Current and future

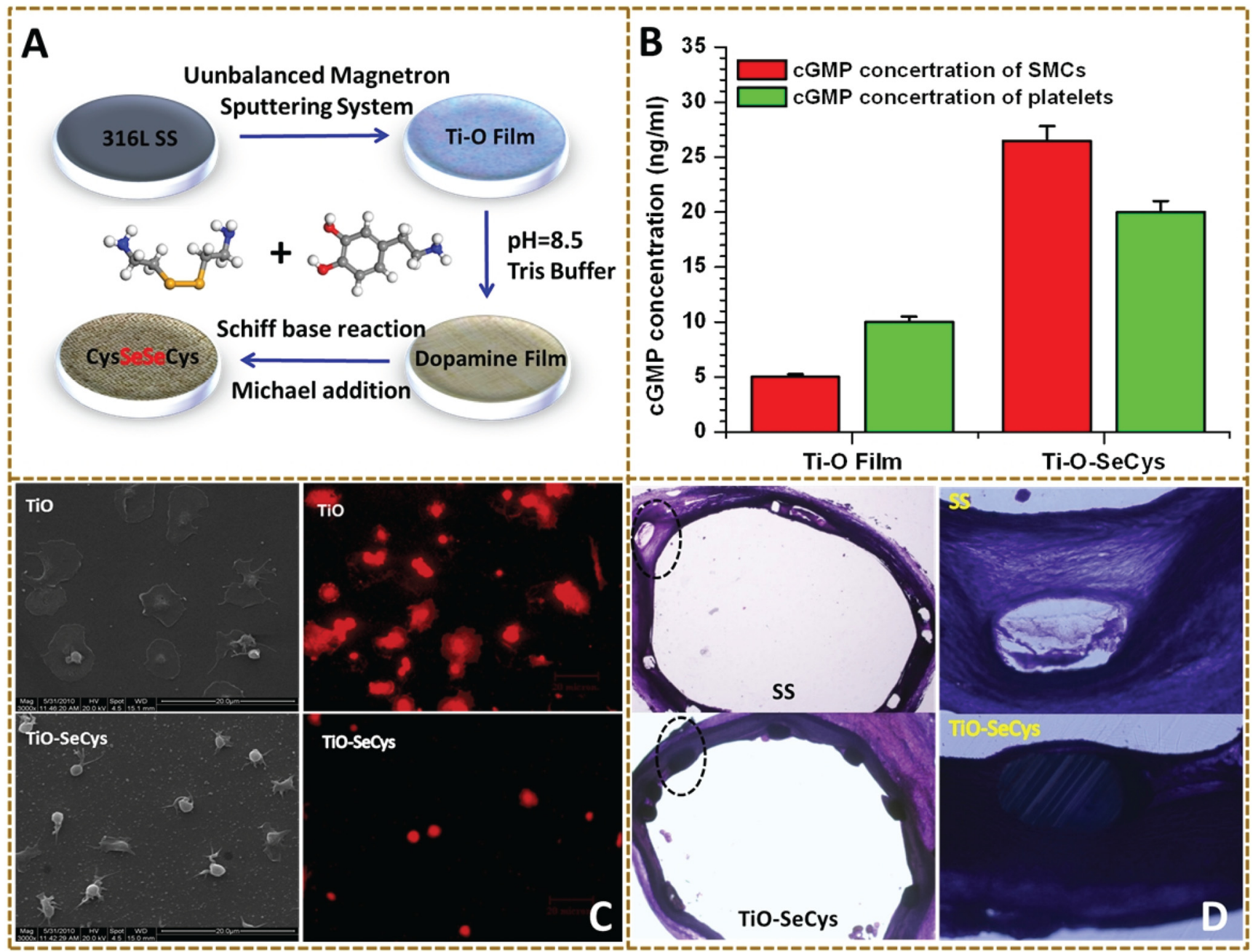

FIG. 8. (a) Schematic diagram of SeCys immobilization on polydopamine modified surface (TiO-SeCys). (b) cGMP concentration of platelets and SMCs on Ti-O film and Ti-O-SeCys samples. (c) Platelets and SMCs adhesion on Ti-O film and Ti-O-SeCys samples. The unactived state platelets and absent adhesion of SMCs on the Ti-O-SeCys samples are shown. (d) Optical photographs of the cross section slices of canine femoral arteries with SeCys functionalized (TiOSeCys) and control (SS) stents. The circle shows the differences in intimal hyperplasia. In the detail magnifications, lumen loss can be easily recognized in the control but TiO-SeCys also keeps patent after two months. 
research on constructing NO releasing surfaces especially stent surfaces will undoubtedly attract scientists, engineers, and clinicians the opportunity to make significant advances in the treatment and prevention of life threatening cardiovascular disorders such as atherosclerosis. ${ }^{98}$

However, an ideal stent surface that could address all the problems does not exist. For each method of multifunctional surfaces, the specific disadvantages and limitations should be carefully considered. Since most of the presented results are based on in vitro or animal in vivo studies, few designs have undergone the clinical evaluation yet. It has not been proven so far, that the multifunctional surface performs well in a complex biological environment. For example, the biostability of the immobilized molecules is a crucial point especially under long-term interactions with various enzymes or other specific molecules. In the case of NO releasing systems, the sufficiency of the NO-donor loading is unclear, or a possible block of the catalytic reaction sites (e.g., biomimetic organoselenium catalyst) can provide challenges in the designing of this surface type. MNPs-assisted stem cell delivery system might avoid the above mentioned disadvantages. Yet, cellular uptake, intracellular trafficking, and cytotoxicity of MNPs as a matter of particle size await further investigation. Also, as the MNPs get systemic distribution, the biosafety of MNPs concerning other vital organs needs to be evaluated. ${ }^{99}$ Thus, there is still a long way before application of these strategies in future stents.

\section{CONCLUSION}

With the objective of developing an innovative cardiovascular stent surface to simultaneously improve hemocompatibility, endothelialization, and antiproliferative properties, multifunctional surfaces were constructed in the author's group via different techniques. Tailoring BMS, DES, and EPC capture stents, different molecules including heparin, gallic acid, and various aptamers became our candidates for novel stent designs. The systematic evaluation in vitro and in vivo encouraged us to deeper exploration of these stent models, and further basic experiments, animal tests, and clinical trials need to be carried out. Also, we are looking forward to the applications of nitric oxide releasing or generating coatings in an ideal stent design, which presents a huge potential in the cardiovascular system. Moreover, there even exists the possibility of healing atherosclerosis due to the diverse functions of nitric oxide. However, despite extensive research to vascular stent for endothelium regenerative purposes, not many biofunctionalized stents have been successfully translated into the clinic. There are difficulties in combining multiple signals to satisfy the microenvironment where stent behaviors, which result in the unsuccessful application in clinic. Therefore, the multifunctional stent surface involved physical, biological, and chemical properties need to be integrated and tuned for better control the different processes of endothelium healing. We believe that our various multifunctional stent surfaces will present good biocompatibility in the ongoing in vivo animal tests and enter clinical trials in the near future. Scientists will spare no effort on improving stent properties, providing systematic evaluation of novel stents combining advanced surface modification equipment and cellular or molecular biological techniques and also in the same time, greatly enhance the safety, cost-effectiveness, and efficacy of vascular stent in applications of cardiovascular tissue regeneration and even promote atherosclerosis healing.

\section{ACKNOWLEDGEMENT}

This work was supported by Key Basic Research Program 2011CB606204, NASC 81271701, 31270020, 31300792, and NSFC Key Project 81330031.

${ }^{1}$ R. M. Bergman and R. M. Nerem, Cardiovasc. Eng. Technol. 1, 4 (2010).

${ }^{2}$ World Health Organization, World Health Statistics 2012 (World Health Organization, 2012).

${ }^{3}$ N. Mackman, Nature 451, 914 (2008).

${ }^{4}$ A. de Mel, C. Bolvin, M. Edirisinghe, G. Hamilton, and A. M. Seifalian, Expert Rev. Cardiovasc. Ther. 6, 1259 (2008).

${ }^{5}$ M. Voegele-Kadletz and E. Wolner, Mater. Sci. Eng., C 31, 1195 (2011).

${ }^{6}$ A. Farb, D. K. Weber, F. D. Kolodgie, A. P. Burke, and R. Virmani, Circulation 105, 2974 (2002).

${ }^{7}$ E. P. McFadden, E. Stabile, E. Regar, E. Cheneau, A. T. Ong, T. Kinnaird, and P. W. Serruys, Lancet, 364, 1519 (2004).

${ }^{8}$ M. Klomp, M. A. Beijk, and R. J. de Winter, Expert Rev. Med. Devices 6, 365 (2009).

${ }^{9}$ S. Nishio, K. Kosuga, K. Igaki, M. Okada, E. Kyo, T. Tsuji, and S. Ikeguchi, Circulation 125, 2343 (2012).

${ }^{10}$ U. Westedt, M. Wittmar, M. Hellwig, P. Hanefeld, A. Greiner, A. K. Schaper, and T. Kissel, J. Controlled Release 111, 235 (2006).

${ }^{11}$ N. M. Pires, B. L. van der Hoeven, M. R. de Vries, L. M. Havekes, B. J. van Vlijmen, W. E. Hennink, and J. W. Jukema, Biomaterials 26, 5386 (2005).

${ }^{12}$ M. Haude, R. Erbel, P. Erne, S. Verheye, H. Degen, D. Böse, and J. Koolen, Lancet 381, 836 (2013).

${ }^{13}$ M. Schinhammer, A. C. Hänzi, J. F. Löffler, and P. J. Uggowitzer, Acta Biomater. 6, 1705 (2010).

${ }^{14}$ P. K. Bowen, J. Drelich, and J. Goldman, Adv. Mater. 25, 2577 (2013).

${ }^{15}$ H. Hermawan, D. Dube, and D. Mantovani, Acta Biomater. 6, 1693 (2010).

${ }^{16}$ B. O'Brien and W. Carroll, Acta Biomater. 5, 945 (2009).

${ }^{17}$ S. Pant, G. Limbert, N. P. Curzen, and N. W. Bressloff, Biomaterials 32, 7755 (2011).

${ }^{18}$ B. Bhargava, I. De Scheerder, Q. B. Ping, H. Yanming, R. Chan, H. Soo Kim, and M. B. Leon, Catheter. Cardiovasc. Interventions 51, 364 (2000).

${ }^{19}$ P. Qi, M. F. Maitz, and N. Huang, Surf. Coat. Technol. 233, 80 (2013).

${ }^{20}$ F. Otsuka, A. V. Finn, S. K. Yazdani, M. Nakano, F. D. Kolodgie, and R. Virmani, Nat. Rev. Cardiol. 9, 439 (2012).

${ }^{21}$ E. A. Vogler and C. A. Siedlecki, Biomaterials 30, 1857 (2009).

${ }^{22}$ C. Sperling, M. Fischer, M. F. Maitz, and C. Werner, Biomaterials 30, 4447 (2009)

${ }^{23}$ E. W. Davie, J. Biol. Chem. 278, 50819 (2003).

${ }^{24}$ C. Werner, M. F. Maitz, and C. Sperling, J. Mater. Chem. 17, 3376 (2007).

${ }^{25}$ M. B. Gorbet and M. V. Sefton, Biomaterials 25, 5681 (2004).

${ }^{26}$ A. Zalewski, Y. Shi, and A. G. Johnson, Circ. Res. 91, 652 (2002).

${ }^{27}$ D. Fukuda and M. Aikawa, Circulation 122, 2005 (2010).

${ }^{28}$ J. I. Koga and M. Aikawa, Vasc. Pharmacol. 57, 24 (2012).

${ }^{29}$ M. S. Caicedo, P. H. Pennekamp, K. McAllister, J. J. Jacobs, and N. J. Hallab, J. Biomed. Mater. Res., Part A 93, 1312 (2010).

${ }^{30}$ N. Kukreja, Y. Onuma, J. Daemen, and P. W. Serruys, Pharmacol. Res. 57, 171 (2008)

${ }^{31}$ G. G. Stefanini and D. R. Holmes, Jr., N. Engl. J. Med. 368, 254 (2013).

${ }^{32}$ N. Huang, P. Yang, Y. X. Leng, J. Y. Chen, H. Sun, J. Wang, and Y. Leng, Biomaterials 24, 2177 (2003). 
${ }^{33}$ P. Qi, Y. Yang, F. M. Maitz, and N. Huang, Chin. Sci. Bull. 58, 4362 (2013).

${ }^{34}$ J. Daemen and P. W. Serruys, Circulation 116, 316 (2007).

${ }^{35}$ D. E. Cutlip, S. Windecker, R. Mehran, A. Boam, D. J. Cohen, G. A. van Es, and P. W. Serruys, Circulation 115, 2344 (2007).

${ }^{36}$ M. A. Costa and D. I. Simon, Circulation 111, 2257 (2005).

${ }^{37}$ M. M. De Villiers, D. P. Otto, S. J. Strydom, and Y. M. Lvov, Adv. Drug Delivery Rev. 63, 701 (2011).

${ }^{38}$ S. Meng, Z. Liu, L. Shen, Z. Guo, L. L. Chou, W. Zhong, and J. Ge, Biomaterials 30, 2276 (2009).

${ }^{39}$ N. M. Luan, Y. Teramura, and H. Iwata, Biomaterials 32, 6487 (2011).

${ }^{40}$ T. G. Kim, H. Lee, Y. Jang, and T. G. Park, Biomacromolecules 10, 1532 (2009).

${ }^{41}$ S. Lu, P. Zhang, X. Sun, F. Gong, S. Yang, L. Shen, and C. Wang, ACS Appl. Mater. Interfaces 5, 7360 (2013).

${ }^{42}$ S. Hossfeld, A. Nolte, H. Hartmann, M. Recke, M. Schaller, T. Walker, and R. Krastev, Acta Biomater. 9, 6741 (2013).

${ }^{43}$ C. M. Jewell, J. Zhang, N. J. Fredin, M. R. Wolff, T. A. Hacker, and D. M. Lynn, Biomacromolecules 7, 2483 (2006).

${ }^{44} \mathrm{G}$. Li, P. Yang, Y. Liao, and N. Huang, Biomacromolecules 12, 1155 (2011).

${ }^{45}$ G. Li, P. Yang, W. Qin, M. F. Maitz, S. Zhou, and N. Huang, Biomaterials 32, 4691 (2011).

${ }^{46}$ G. Li, P. Yang, X. Guo, N. Huang, and R. Shen, Cytokine 56, 208 (2011).

${ }^{47}$ J. L. Chen, Q. L. Li, J. Y. Chen, C. Chen, and N. Huang, Appl. Surf. Sci. 255, 6894 (2009).

${ }^{48}$ K. Zhang, J. A. Li, K. Deng, T. Liu, J. Y. Chen, and N. Huang, Colloids Surf., B 108, 295 (2013).

${ }^{49}$ M. Avci-Adali, G. Ziemer, and H. P. Wendel, Biotechnol. Adv. 28, 119 (2010).

${ }^{50}$ T. Asahara, T. Murohara, A. Sullivan, M. Silver, R. van der Zee, T. Li, and J. M. Isner, Science 275, 964 (1997).

${ }^{51}$ M. A. Beijk, M. Klomp, N. J. Verouden, N. van Geloven, K. T. Koch, J. P. Henriques, and R. J. de Winter, Eur. Heart J. 31, 1055 (2010).

${ }^{52}$ Q. Lin, X. Ding, F. Qiu, X. Song, G. Fu, and J. Ji, Biomaterials 31, 4017 (2010).

${ }^{53}$ Q. L. Li, N. Huang, C. Chen, J. L. Chen, K. Q. Xiong, J. Y. Chen, and X. Liang, J. Biomed. Mater. Res., Part A 94, 1283 (2010).

${ }^{54}$ S. Liu, T. Liu, J. Chen, M. Maitz, C. Chen, and N. Huang, J. Biomed. Mater. Res., Part A 101A, 1144 (2012).

${ }^{55}$ H. P. Wendel, M. Avci-Adali, and G. Ziemer, Int. J. Cardiol. 145, 115 (2010).

${ }^{56}$ M. Avci-Adali, H. Stoll, N. Wilhelm, N. Perle, C. Schlensak, and H. P. Wendel, Pathobiology 80, 176 (2013).

${ }^{57}$ J. M. Lee, W. Choe, B. K. Kim, W. W. Seo, W. H. Lim, C. K. Kang, and H. S. Kim, Biomaterials 33, 8917 (2012).

${ }^{58}$ A. N. Veleva, D. E. Heath, S. L. Cooper, and C. Patterson, Biomaterials 29, 3656 (2008).

${ }^{59}$ J. Hoffmann, A. Paul, M. Harwardt, J. Groll, T. Reeswinkel, D. Klee, and H. P. Wendel, J. Biomed. Mater. Res., Part A 84, 614 (2008).

${ }^{60}$ Q. Li, G. Tang, S. Xue, X. He, P. Miao, Y. Li, and G. Y. Yang, Biomaterials 34, 4982 (2013).

${ }^{61}$ J. Riegler, A. Liew, S. O. Hynes, D. Ortega, T. O’Brien, R. M. Day, and M. F. Lythgoe, Biomaterials 34, 1987 (2012).

${ }^{62}$ J. L. Chen, N. Huang, B. L. Ma, M. F. Maitz, J. Wang, J. A. Li, and X. Liu, ACS Appl. Mater. Interfaces 5, 5976 (2013).

${ }^{63}$ B. Polyak, I. Fishbein, M. Chorny, I. Alferiev, D. Williams, B. Yellen, and R. J. Levy, Proc. Natl. Acad. Sci. 105, 698 (2008).

${ }^{64}$ J. McLean, Circulation 19, 75 (1959).
${ }^{65}$ I. Capila and R. J. Linhardt, Angew. Chem. Int. Ed. 41, 390 (2002).

${ }^{66}$ R. J. Linhardt, S. Murugesan, and J. Xie, Curr. Top. Med. Chem. 8, 80 (2008).

${ }^{67}$ M. F. Maitz, U. Freudenberg, M. V. Tsurkan, M. Fischer, T. Beyrich, and C. Werner, Nat. Commun. 4, 2168 (2013).

${ }^{68}$ J. A. Beamish, L. C. Geyer, N. A. Haq-Siddiqi, K. Kottke-Marchant, and R. E. Marchant, Biomaterials 30, 6286 (2009).

${ }^{69}$ Z. Yang, Q. Tu, J. Wang, and N. Huang, Biomaterials 33, 6615 (2012).

${ }^{70}$ Z. Yang, J. Wang, R. Luo, M. F. Maitz, F. Jing, H. Sun, and N. Huang, Biomaterials 31, 2072 (2010).

${ }^{71}$ T. Liu et al., Acta Biomater. 10, 1940 (2013).

${ }^{72}$ F. Gong, X. Cheng, S. Wang, Y. Zhao, Y. Gao, and H. Cai, Acta Biomater. 6, 534 (2010).

${ }^{73}$ E. M. Stewart, X. Liu, G. M. Clark, R. M. I. Kapsa, and G. G. Wallace, Acta Biomater. 8, 194 (2012).

${ }^{74}$ C. T. Ho, Q. Chen, H. Shi, K. Q. Zhang, and R. T. Rosen, Prev. Med. 21, 520 (1992).

${ }^{75}$ R. Abu-Amsha Caccetta, V. Burke, T. A. Mori, L. J. Beilin, I. B. Puddey, and K. D. Croft, Free Radicals Biol. Med. 30, 636 (2001).

${ }^{76}$ B. R. You and W. H. Park, Toxicol. In Vitro 24, 1356 (2010).

${ }^{77}$ Y. He, J. Wang, W. Yan, and N. Huang, RSC Adv. 4, 212 (2014).

${ }^{78}$ S. Chen, X. Li, Z. Yang, S. Zhou, R. Luo, M. F. Maitz, and N. Huang, Colloids Surf., B 113, 125 (2014).

${ }^{79}$ Z. Yang, Y. Yang, W. Yan, Q. Tu, J. Wang, and N. Huang, ACS Appl. Mater. Interfaces 5, 10495 (2013).

${ }^{80}$ Z. Yang, K. Xiong, P. Qi, Y. Yang, Q. Tu, J. Wang, and N. Huang, ACS Appl. Mater. Interfaces 6, 2647 (2014).

${ }^{81}$ L. J. Ignarro, G. Cirino, A. Casini, and C. Napoli, J. Cardiovasc. Pharm. 34, 879 (1999).

${ }^{82}$ F. Murad, Angew. Chem. Int. Ed. 38, 1856 (1999).

${ }^{83}$ D. A. Riccio and M. H. Schoenfisch, Chem. Soc. Rev. 41, 3731 (2012).

${ }^{84}$ A. W. Carpenter and M. H. Schoenfisch, Chem. Soc. Rev. 41, 3742 (2012).

${ }^{85}$ W. Cai, J. Wu, C. Xi, A. J. Ashe, and M. E. Meyerhoff, Biomaterials 32, 7774 (2011).

${ }^{86}$ K. M. Naseem, Mol. Aspects Med. 26, 33 (2005).

${ }^{87}$ M. Kushwaha, J. M. Anderson, C. A. Bosworth, A. Andukuri, W. P. Minor, J. R. Lancaster, and H. W. Jun, Biomaterials 31, 1502 (2010).

${ }^{88}$ W. Cai, J. Wu, C. Xi, and M. E. Meyerhoff, Biomaterials 33, 7933 (2012).

${ }^{89}$ C. Napoli, F. de Nigris, S. Williams-Ignarro, O. Pignalosa, V. Sica, and L. J. Ignarro, Nitric Oxide 15, 265 (2006).

${ }^{90}$ P. R. Kvietys and D. N. Granger, Free Radicals Biol. Med. 52, 556 (2012).

${ }^{91}$ A. Andukuri, Y. D. Sohn, C. P. Anakwenze, D. J. Lim, B. C. Brott, Y. S. Yoon, and H. W. Jun, Tissue Eng., Part C 19, 375 (2012).

${ }^{92}$ M. C. Jen, M. C. Serrano, R. van Lith, and G. A. Ameer, Adv. Funct. Mater. 22, 239 (2012).

${ }^{93}$ N. Naghavi, A. de Mel, O. S. Alavijeh, B. G. Cousins, and A. M. Seifalian, Small 9, 22 (2013).

${ }^{94}$ S. Hwang, W. Cha, and M. Meyerhoff, Angew. Chem. 118, 2811 (2006).

${ }^{95}$ W. Cha and M. E. Meyerhoff, Biomaterials 28, 19 (2007).

${ }^{96}$ Y. Weng, Q. Song, Y. Zhou, L. Zhang, J. Wang, J. Chen, and N. Huang, Biomaterials 32, 1253 (2011).

${ }^{97}$ L. J. Ignarro, Angew. Chem. Int. Ed. 38, 1882 (1999).

${ }^{98}$ C. Cheng, D. Tempel, R. van Haperen, A. van der Baan, F. Grosveld, M. J. Daemen, and R. de Crom, Circulation 113, 2744 (2006).

${ }^{99}$ L. H. Reddy, J. L. Arias, J. Nicolas, and P. Couvreur, Chem. Rev. 112, 5818 (2012). 